\title{
Optimization of the reliability of heat supply with an active consumer
}

\author{
Ivan Postnikov ${ }^{1, *}$, and Andrey Penkovsky ${ }^{1}$ \\ ${ }^{1}$ Melentiev Energy Systems Institute of the Siberian Branch of the Russian Academy of Sciences, \\ 664033, Lermontova str., 130, Irkutsk, Russia
}

\begin{abstract}
The paper formulates the problem of ensuring the reliability of the heat supply system due to the optimal distribution of reliability parameters in the system, including taking into account capabilities of an active consumer to replace part of the load with own sources of thermal energy. Methods and models for solving this problem are proposed, which are based on the theory of random processes, the theory of hydraulic circuits, and the basic laws of heating. Results of the computational experiment based on the developed methodical apparatus are presented. The possible economic effect of using an active consumer for solving the describe problem is also reviewed in the paper.
\end{abstract}

\section{Introduction}

One of the main principles of the modern paradigm of building energy systems is the strengthening the role of the consumer in energy supply. This principle is implemented within the framework of the concept of an active consumer (AC), whose functions consist in regulating and optimizing the schedule of personal consumption from their own sources and energy accumulators in order to increase the efficiency and reliability of both the consumer and the entire power system.

A number of studies [1-7] deal with various aspects of the functioning and management of AP regimes (in the English-language literature - prosumer) as part of power supply systems. Significantly, the methodological issues of the AC operation in district heating systems are raised $[8,9]$. At the same time, these technologies are also relevant for the heat supply systems (HSS), which are the largest consumers of fuel and energy.

With the functioning of modern HSS, especially in conditions of increasing thermal loads, there are a number of problems associated with meeting the requirements focused on volumes and reliability of providing consumers with thermal energy. The creation of an AC in the system (hereinafter referred to an active consumer of thermal energy) with own sources of heat energy $(\mathrm{SH})$ provides an additional reserve of power and time, which reduces the burden on the centralized SH and thereby increases the quality and reliability of heat supply to both the consumers themselves and the system functioning as a whole.

Thus, one of the key objectives of the AC in the HSS is to increase the reliability of its operation. The methodological tasks that need to be solved to achieve this goal are, in fact,

\footnotetext{
*Corresponding author: postnikov@isem.irk.ru
} 
traditional for analyzing and synthesizing the reliability of energy systems in general and in the HSS, in particular. At the same time, the inclusion of AC in the HSS makes new aspects to these tasks and requires the use of new methodological approaches for their solution.

\section{Statement of the problem}

In the development of methods for analyzing and optimizing the HSS reliability with the participation of the AC, there are two main areas of research. The first is related to the analysis and optimization of the consumers' heat supply reliability, taking into account the functions of the AC in the system, provided that the number, nodes, and capacities of the AP's own sources are specified. The second direction is the task of determining the number, locations. and power of the AC, along with other ways to ensure system reliability.

In this paper, we consider the first reliability problem, which consists in determining such a structure (distribution by system) of the values of the reliability parameters of the HSS elements (failure and recovery intensities), which provides the required level of reliability of heat supply to consumers, including the AC taking into account their additional reserve, with minimum costs to achieve these parameters in the range of their admissible values.

To assess the level of reliability of heat supply to consumers, two main reliability indicators (RI) have been adopted [10]: (a) the probability of failure-free operation $R j$ (FBD) and (b) the availability factor $K j$ (AF) being determined for each consumer $j \in J$, where $J$ is the set of system consumers (including ACs, their availability). The normative values of these RIs are denoted by $R o j$ and $K o j$.

Concerning these RIs, the problem is solved by the method consisting of four stages: 1) determining the relationship between the integral reliability parameters of the HSS elements; 2) simulating post-emergency HSS modes; 3) modeling of AC functions; 4) formalizing the problem of determining the optimal parameters of HSS elements reliability.

\section{Mathematical formalization of the problem of optimizing the reliability parameters of the HSS elements taking into account functions of the active consumer}

The solution of the problem posed in the previous section is based on the concept of an integral parameter of reliability of the system elements. The integral parameter of elements reliability means the intensity of their failure or recovery, which has the same (averaged) value for these elements, at which the required level of RI is provided. To determine these parameters, formulas were used for calculating the nodal RI of the HSS [10], the Rossander equation determining the annual graph of the thermal loads of consumers [11], as well as some basic patterns of heat and thermal processes in heat supply [12].

Optimization of reliability parameters of HSS elements, taking into account the functions of the active consumer, is formulated as follows:

To find:

$$
F_{\text {obj }}=\sum_{n \in N} f_{n \lambda}\left(\lambda_{n}\right)+\sum_{n \in N} f_{n \mu}\left(\mu_{n}\right) \rightarrow \min
$$

under the following conditions and limitations:

$$
\bar{\lambda}_{j}=\frac{1}{\tau_{\mathrm{o}}}\left[\ln \left(1 / R_{\mathrm{o} j}\right)\right] \times\left(1-N_{s}\left(1-K_{\mathrm{o} j}\right) / \sum_{s \in E} L_{s}^{1 / \sigma_{j}}\right)^{-1} \times\left(\sum_{s \in E} M_{s}^{1 / \sigma_{j}}\right)^{-1} ;
$$




$$
\begin{aligned}
& L_{s}=\frac{1}{1-\omega_{j}}\left[1-\frac{1}{q_{\mathrm{o} j}}\left(q_{s j}+\varphi_{j} t_{s j}-\varphi_{j} \frac{C_{1}-C_{2} \exp B_{j}}{C_{3}\left(1-\exp B_{j}\right)}\right)\right] \\
& M_{s}=\frac{1}{1-\omega_{j}}\left(1-\bar{q}_{s j}+\frac{\varphi_{j} t_{s j}}{q_{\mathrm{o} j}}-\varphi_{j} \frac{C_{1}-C_{2} \exp B_{j}}{C_{3} q_{\mathrm{oj}}\left(1-\exp B_{j}\right)}\right) \\
& C_{1}=t_{\mathrm{oj}}\left(1-\bar{q}_{s j}\right), C_{2}=t_{j \min }-t_{\mathrm{oj}} \bar{q}_{s j}, C_{3}=1-\bar{q}_{s j} ; \\
& \bar{q}_{s j}=q_{\mathrm{o} j} /\left(q_{s j}^{\mathrm{sys}}+q_{s j}^{\prime}\right), s \in E, j \in J \\
& B_{j}=1 /\left[\left(\varepsilon_{j}+\Delta \varepsilon_{j}\right) \bar{\mu}_{j}\right], \quad j \in J \\
& \bar{\lambda}_{j} \sum_{s \in E} p_{s}=\sum_{n \in N} \sum_{s \in E(n)} \lambda_{n} p_{s}, j \in J \\
& \bar{\mu}_{j} \sum_{s \in E} p_{s}=\sum_{n \in N} \sum_{s \in E(n)} \mu_{n} p_{s}, j \in J \\
& p_{s}\left(\sum_{n \in N(s)} \lambda_{n}+\sum_{n \in N(s)} \mu_{n}\right)=\sum_{z \in E(s)}\left(\sum_{n \in N(z)} p_{z} \lambda_{n}+\sum_{n \in N(z)} p_{z} \mu_{n}\right), s \in E \\
& \mathbf{A} \mathbf{x}_{s}=\mathbf{q}_{s}, s \in S \\
& \overline{\mathbf{A}}_{s}^{\mathrm{T}} \mathbf{p}_{s}=\mathbf{h}_{s}-\mathbf{H}_{s}, s \in S \\
& \mathbf{S} \mathbf{X}_{S} \mathbf{x}_{S}=\mathbf{h}_{s}, s \in S \\
& \lambda_{n}^{\min } \leq \lambda_{n} \leq \lambda_{n}^{\max }, n \in N \\
& \mu_{n}^{\min } \leq \mu_{n} \leq \mu_{n}^{\max }, n \in N
\end{aligned}
$$

Here: $f_{n \lambda}\left(\lambda_{n}\right), f_{n \mu}\left(\mu_{n}\right)$ are the cost functions to ensure the elements' reliability parameters (intensity of their failure and recovery), respectively, rubles; $\bar{\lambda}_{j}$ and $\bar{\mu}_{j}$ are the integral failure and recovery rates for the consumer $j$, respectively, $1 / \mathrm{h} ; \tau_{\mathrm{o}}$ is the moment of time corresponding to the total number of hours of the heating period, h; Ns is the number of states of the system; $s$ is the system status number; $E$ is the set of states of the system; $\omega_{j}, \sigma_{j}$ are the coefficients of the graph unevenness in terms of the thermal load of the consumer $j[11] ; \varphi_{j}$ is the coefficient of specific heat losses for the consumer $j$, GJ / $\left(\mathrm{h}^{\circ} \mathrm{C}\right)$; 
$t_{\mathrm{sj}}$ is the current (actual) internal air temperature for the consumer $j$ in the system state $s,{ }^{\circ} \mathrm{C}$; $\bar{q}_{s j}$ stands for relative supply of thermal energy to the consumer $j$ in the state $s$ of the system, $\mathrm{GJ} / \mathrm{h} ; t_{o j}$ means the design temperature of internal air for the consumer $j,{ }^{\circ} \mathrm{C} ; t_{j} \min$ is the minimum permissible temperature of the indoor air for the user $j,{ }^{\circ} \mathrm{C} ; q_{s j}^{\mathrm{sys}}$ stands for part of the thermal load of the consumer $j$ being covered from the system (centralized SH) in the state $s$ of the system, GJ/h; $q_{s j}^{\prime}$ id the performance of the customer's own $\mathrm{SH} j$ (for AC) in the state $s$ of the system, $\mathrm{GJ} / \mathrm{h} ; \varepsilon_{j}$ is the coefficient of thermal accumulation of the consumer $j, \mathrm{~h} ; \Delta \varepsilon_{j}$ stands for additional passive temporary reserve of the $\mathrm{AC}$ (due to use of own $\mathrm{SH}$ and/or heat accumulators), $\mathrm{h} ; L_{s}, M_{s}, C_{1}, C_{2}, C_{3}, B_{j}$ are the accepted abbreviations; $p_{s}$ is the probability of the state $s$ of the system; $n$ is the number of the system element; $N$ is the set of elements of the system; $E(n)$ is a subset of the states of the system, from which a transition is possible, associated with the failure or restoration of the element $n ; \lambda_{n}, \mu_{n}$ is the intensity of failure and recovery of the element $n, 1 / \mathrm{h} ; p_{z}$ is the probability of the state of the system (separation of states by $s$ and $z$ is necessary for recording the system of equations of a random process); $N(s)$ is the subset of system elements whose failure or recovery corresponds to a direct transition of the system from state $s$ to some other state $s ; N(z)$ is a subset of the elements of the system whose failure or recovery corresponds to a direct transition of the system from the state $z$ to some other state $s ; E(s)$ is a subset of the states of the system, from which a direct transition to the state $s$ is possible; $A_{s}$ is the matrix of connections of linearly independent nodes of the thermal network (TN) under the conditions of the emergency state $s$ (taking into account the failure of any element); $\overline{\mathbf{A}}_{S}^{\mathrm{T}}$ stands for the complete transposed matrix of connections of nodes and branches of the TN under conditions of emergency state $s[13] ; x_{\mathrm{s}}$ is the vector of the coolant flow on the branches of the network under the conditions of the emergency state $s, \mathrm{t} / \mathrm{h}[13] ; q_{s}$ is the vector of expenses in network nodes under conditions of emergency state $s, \mathrm{t} / \mathrm{h}[13] ; p_{s}$ is the vector of node pressures of the thermal network under the conditions of the system's emergency state, $\mathrm{mm} \mathrm{Hg}$. [13]; $h_{s}$ is the vector of losses of heads on the branches of the network under the conditions of the emergency state $s, \mathrm{~mm} \mathrm{Hg}$. [13]; $H_{\mathrm{s}}$ is the vector of active heads on the sources under the conditions of the emergency state $s, \mathrm{~mm} \mathrm{Hg}$. [13]; $S, X_{s}$ are the diagonal matrices of the coefficients of the hydraulic resistance of the branches, made up of the hydraulic resistances of the branches and the absolute values of the charges on them [13]; $\lambda_{n}^{\min }, \lambda_{n}^{\max }$ - limitations on technically possible values of failure rates of elements, $1 / \mathrm{h} ; \mu_{n}^{\min }, \mu_{n}^{\max }$ - restrictions on technically possible values of the recovery rates of elements, $1 / \mathrm{h}$.

The objective function $F_{\text {obj }}$ of the HSS reliability optimization in accordance with the task is expressed by the sum of the cost functions for providing the values of the reliability parameters of its elements (1). Determining the type and parameters of these functions is a separate technical and economic task, which is carried out by methods of approximating data on the cost of installation of equipment with various reliability characteristics, its reservation, the creation of emergency and recovery services (ERS) and other ways to ensure the HSS reliability. The indicator $q^{\prime}$ sj, which determines the AC reserve, can be fixed and correspond to the calculated (required) value of the performance of the own $\mathrm{SH}$ of the AC; it may be variable and take into account the failures of their elements, leading to a decrease in their performance, while a number of system states must be supplemented with appropriate failures. In this case, the decision should be made on the basis of analyzing the initial characteristics of the equipment reliability: if the failure flow rate for the $\mathrm{SH}$ 
equipment of the AC is significantly lower than for the HSS system equipment, than SH (AC) failures can be ignored, and $q^{\prime}$ sj can be fixed in accordance with the required productivity at the given emergency heat supply of the consumer.

The probabilities of the system states are determined from the solution of the Markov process equations (10), describing the sequence and structure of events characterizing the HSS functioning. Markov models are a valid and universal apparatus for assessing the reliability of recoverable systems [14-17]. The validity of applying the stationary Markov model, as well as other aspects of using the apparatus of Markov random processes in reliability problems of HSS, are considered in more detail in the papers [14, 18-22].

The post-emergency hydraulic regime is determined by the flow distribution in the TN, which is calculated by the methods of the theory of hydraulic circuits [13] using the flowdistribution model presented in the nodal matrix form (11), (12), and (13). Simulation of an emergency in some state $S$ of the system is performed by excluding the element from the design scheme (whose failure corresponds to this state).

The mathematical method of solving the formulated problem is determined mainly by the form of the cost functions (1). Usually, using the approximation methods, these functions can be represented in the form of power dependencies.

\section{Computational experiment and analysis of results}

The computational experiment is carried out on the basis of the HSS scheme shown in Figure 1 and consisting of two heat sources (SH1, SH2), a ring TN of 18 sections, seven consumers (nodes 1-7), one of which is active (AC 6) with a load of $400 \mathrm{GJ} / \mathrm{h}$ and the SH capacity of $200 \mathrm{GJ} / \mathrm{h}$. The enlarged elemental scheme for both centralized SH is shown in Figure 2 and consists of the following basic elements: a boiler 19, a turbine 20, network heaters 21 and 23, and network pumps 22 and 24. The indicated item numbers correspond to SH1, they are assigned in order from 25 to 30 for $\mathrm{SH} 2$.

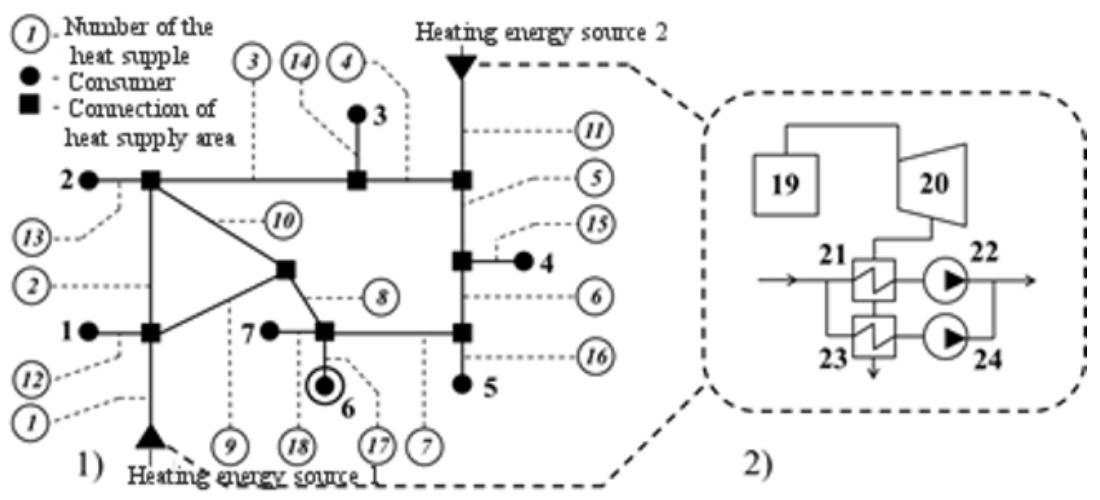

Fig. 1. The HSS test scheme: 1) a general HSS scheme; 2) a simplified SH scheme.

The designed HSS test scheme is formed according to the integrated approach to the investigation of the heat supply reliability [18-20] in the form of a single structure that unites elements of the SH and TN schemes and is shown in Figure 2. The elements 1-18 are parts of the network, while the elements 19-30 correspond to SH1 and SH2. Simulation of a random process of HSS functioning is carried out under the following conditions: each element can be in an efficient and faulty state, and the event flow in the HSS is the simplest. The formation of a plurality of states is limited to considering the states of joint failure of no more than two elements from different subsystems of the HSS (TN, SH1 and SH2). Thus, a 
lot of states are formed by failure states of each element of the TN, SH1, SH2, and combinations of these states: $\mathrm{TN}+\mathrm{SH} 1, \mathrm{TN}+\mathrm{SH}$, and $\mathrm{SH} 1+\mathrm{SH} 2$. An oriented graph reflecting the structure of the HSS states is shown in the abbreviated form in Figure 2. The status numbers on the graph correspond to the failed elements (the elements 1-18 are the network sections, while the elements 19-30 correspond to the sources). The Markov random process satisfying given conditions is described by a system of 283 linear equations of the form (14). Determining the levels of coolant supply to consumers in different states of HSS is carried out on the basis of multivariate calculations of flow distribution in the TN with allowance for SH equipment failures using the models (9), (10), and (11). The results of calculating the probabilities of system states and post-emergency hydraulic regimes due to a large amount of data are not given.

Optimization of the HSS reliability parameters is carried out under the condition that the following normative values of the RI nodes are fulfilled [10]: $K_{o j}=0.97$ and $R_{o j}=0.905$. The following ranges of possible values of the optimized reliability parameters of the HSS elements are adopted: for failure rates, it is from 0.0002 to $0.00251 / \mathrm{h}$; for the recovery intensity, it is from 0.007 to $0.091 / \mathrm{h}$.

The cost functions for increasing the reliability of the HSS elements that form the objective function (15) were obtained on the basis of analyzing and processing data on the composition and unit cost of the reserve elements of the HSS, technical and economic indicators for the ERS creation and maintenance of different composition [10]. As a result, with the help of approximation methods, necessary dependences of costs on reliability parameters in the form of power functions were obtained
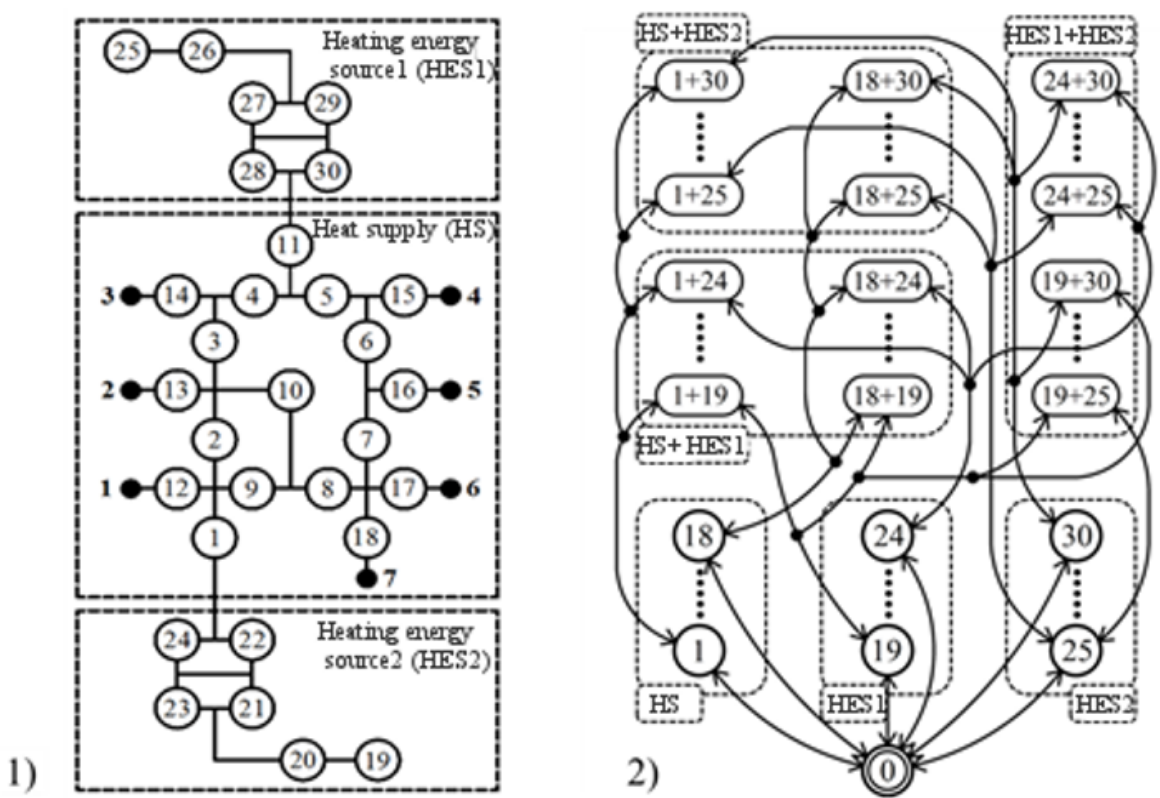

Fig. 2. Simulation of random process in the HSS functioning: 1) the design scheme for reliability analysis; 2) the state graph of the system. 


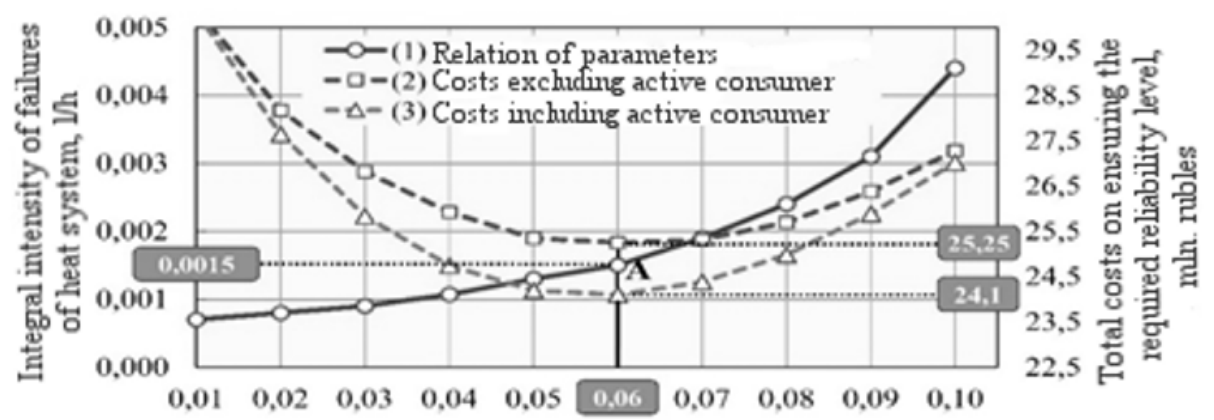

Integral intensity of restoration of heat system elemtnts, lh

Fig. 3. Searching for the optimal ratio of the HSS elements' integral reliability parameters with the AC participation.

The Figure 3 illustrates searching for the optimal ratio of the HSS elements' the integral reliability parameters of corresponding to the minimum costs for ensuring reliability by the system when performing the required PN. The diagram shows three graphs: (a) the line 1 represents the relationship between the integral parameters of the reliability of the elements; (b) the line 2 and (c) the line 3 reflect changexs in the cost of ensuring the required level of reliability of heat supply, depending on the ratio of the integral parameters of the elements' reliability without taking into account the SH6 functioning, respectively. The solution obtained (point A) corresponds to the cost of ensuring reliability in the amount of 25.25 million rubles without participation of SH of an AC 6 and 24.1 million rubles with participation. The economic effect from applying one $\mathrm{AC}$ in the system under consideration is 1.15 million rubles, or $4.5 \%$. In this case, the integral reliability parameters take the following values: the failure rate is $0.00151 / \mathrm{h}$, the recovery rate is $0.061 / \mathrm{h}$.

\section{Conclusion}

In summary, the paper has formulated the problem of ensuring the HSS reliability, including taking into account the functions of AC as ways of additional reserve of heat capacity and time at the expense of own SH. To solve this problem, we used nodal RIs, the apparatus of the Markov random process, models of the theory of hydraulic circuits, as well as some basic laws of heat exchange and thermophysical processes in heat supply. The main advantage of the proposed methodology consists in combining directions to reduce failures and increase the recoverability of elements in a single procedure for finding reliability parameters, which allows the most rational distribution of the total potential for improving the reliability of the HSS elements.

The research was performed at Melentiev Energy Systems Institute SB RAS under the support of Russian Science Foundation (Grant №17-19-01209)

\section{References}

1. Ni Zhang, Yu Yan, Wencong Su, Appl. En., 154, 471-479 (2015)

2. Luka Perkovic, Hrvoje Mikulcic, Neven Duic, Journal of Cleaner Production, xxx, 112 (2016)

3. Hongming Yang, Tonglin Xiong, Jing Qiu et al, Appl. En., 167, 353-365 (2016) 
4. Rehman Zafar, Anzar Mahmood, Sohail Razzaq et al. Prosumer based energy management and sharing in smart grid. Renewable and Sustainable Energy Reviews (in press 2017)

5. Stig Odegaard Ottesen, Asgeir Tomasgard, Stein-Erik Fleten, Energy, 94, 828-843 (2016)

6. Dimitrios J. Vergados, Ioannis Mamounakis, Prodromos Makris, Emmanouel Varvarigos, Sustainable Energy: Grids and Networks, 7, 90-103 (2016)

7. Lakshmi Prakash, Sugatha Kumari P.R, Sharanya Chandran et al, Procedia Technology, 21, 338-344 (2015)

8. Lisa Brange, Jessica Englund, Patrick Lauenburg, Appl. En., 164, 492-500 (2016)

9. Lisa Brand, Alexandra Calvén, Jessica Englund et al., Appl. En., 129, 39-48 (2014)

10. E. V. Senna, A. V. Smirnov, A. A. Ionin, Reliability of heat supply systems (Nauka, Novosibirsk, 2000)

11. E. V. Sennova, V. G. Sidler, Mathematical modeling and optimization of developing heat supply systems (Nauka, Novosibirsk, 1985)

12. V. Ya. Sokolov, district heating and heat networks (Publisher MEI, Moscow, 1999)

13. A. P. Merenkov, V. Ya. Khaselev, Theory of hydraulic vhains (Nauka, Moscow, 1985)

14. Yu. N. Rudenko, Reference book on general models of analysis and synthesis of reliability of energy systems (Energoatomizdat, Moscow, 1994)

15. A. A. Chervonyi, V. I. Lukyashchenko, Reliability of complex systems (Mechanical Engineering, Moscow, 1976)

16. A. M. Polovko, S. V. Gurov, Fundamentals of reliability theory (BHV-Petersburg, SPb., 2006)

17. V. A. Kashtanov, A. I. Medvedev, Theory of reliability of complex systems (Fizmatlit, Moscow, 2010)

18. V. A. Stennikov, I. V. Postnikov, Proceedings of the Russian Academy of Sciences. Power Engineering, 2, 107-121 (2011)

19. V. A. Stennikov, I. V. Postnikov, Power Technology and Engineering, 47(6), 446-453 (2014)

20. V. A. Stennikov, I. V. Postnikov, Sustaining power resources through energy optimization and engineering (Engineering Science Reference, Hershey, PA, 2016)

21. I. V. Postnikov, V. A. Stennikov, E. E. Mednikova, A. V. Penkovskii, Energy Procedia, 105, 3083-3088 (2017)

22. E. E. Mednikova, V. A. Stennikov, I. V. Postnikov, Energy Procedia. 105, 3152-3157 (2017) 